\title{
Impact of several parameters on accuracy RFID speed measurement system
}

\author{
Laila Abouzaid $^{1}$, Ahmed Errami $^{1}$, Othmane Benhmammouch ${ }^{1,2}$ \\ ${ }^{1}$ RTSE Hassan II University, ENSEM-Casablanca, Morocco \\ ${ }^{2}$ International University of Casablanca, Morocco
}

\begin{abstract}
Radio Frequency Identification (RFID) is a rapidly growing automatic wireless data collection technology, this technology is applied in a variety of applications, spanning from Supply chain, payments, Race-timing, to transportation. Recently, this technology is becoming increasingly present in some new fields. In this paper we focus on the RFID speed monitoring system. In this effect, a method for verification of speed accuracy is proposed. The proposed method can illustrate the error introduced by each parameter contribute to calculate speed of vehicle using RFID speed system.
\end{abstract}

\section{Introduction}

"Traffic accidents are predicted to become the second leading cause of the death in developing countries by the years 2020" [1]

At Over 1, 2 million human deaths every year on the world's, due to road traffic crashes, and between 20 and 50 million sustain non-fatal injuries. Unfortunately, for almost all countries, in three out of four cases the deceased persons affect young adults who involve excessively in economically productive activity [2].

There are numerous contributory causes of road traffic accidents, which fall into three categories: (1) Meteorological factors caused by the snow, rain or sleet, can provide poor visibility. (2) Technical factors include generally vehicle status and road infrastructures. (3) Human factors, is concerned the both drivers and road users; which appear in all other causes and represent $90 \%$ of fatal crashes. Alcohol, drugs, fatigue, the use of Mobile Phone, not stopping at red lights and breaking the speed limit, are contributory factors that lead to road accidents. However, it is estimated that over $40 \%$ of fatal crashes are caused by excessive or inappropriate speed $[1,3,4]$.

The road safety strategy, in all the countries propose a plan to achieve a reduction in road deaths and serious injuries, the main action strategy to reduce a numbers of accidents linked to speeding, setting out the generalization of speedometers, in the all roads to control the speed with appropriate sanctions [5]. For that reason, this paper will focus on RFID speed measurement systems in order to examine the factors that influence the precision of the system cited above.

The remainder of this paper is structured as follows: Section 2, gives an overview of widespread speedometers, section 3, describes the RFID speed monitoring system, followed by a problem of precision provided by this system, section 4 explains the factors that contribute in this problem and the influence of these parameters on average speed is presented in section 5 .

\section{Overview of Speed Measurement Systems}

The ubiquitous systems of vehicle's speed on the road are mainly split in two categories: Speed detection with Doppler Effect and laser speed detectors (LIDAR). The first type operates according to Doppler shift which based on the change of frequency of an electromagnetic or sound waves emitted by a fixed source and reflected by the moving objects of interest, this frequency is proportional to the speed of the moving object. On the other hand the principle of the second type requires the determination of the distance between the radar and target; is given by the measurement of the delay between the instant of transmission of a pulse and the instant of detection of the reflected pulse, knowing the speed of light. The variation of the distance obtained by the emission of a periodic pulse burst used to deduce target speed $[6,7]$. 
However, the two techniques mentioned earlier plagued by the difficulty to identify vehicles exceeding the speed limit.

Various solutions have been proposed over the years to solve the problem posed, thus afford to measure the vehicle speed. One possible solution based on the use of a coupling between Doppler radar or the laser detector with imaging device configured to capture at least one image or multiple images. Evidently, the acquired scene image offers the license plate number of the vehicle and/or identification of the driver face; in case of infringement, these data are then used to identify the driver and vehicle. However, the image taken in bad weather conditions or the presence of obstacles may increase the risk to recognize vehicles in cases of violations.

There are well-known solutions, resorts to the use of imaging devices which can be generate traffic video and/or capture images whose purpose is to determine and identify vehicles exceeding the speed limit. Unfortunately, the collection of information by Camera video-based speed enforcement system lead to a huge storage of the acquired data, which requires a complicated procedure for pre-process to recognize the license plate number and other information is needed [8,9].

The aforementioned drawbacks are substantially eliminated by the proposed system, in which the speed measuring of a vehicle and identification of this target are performed using the RFID technology and the Doppler Effect. The documents [10] ;exhibit this system that has as principle the simultaneous combination of the acquisition of information relating to the vehicle first determining the speed of the latter through a tag attached to the vehicle. The calculation of the speed is done by using the frequency shift by Doppler Effect extract, from the signals transmitted and received between the RFID reader and the RFID tag. According to the above technology, the work [11] present a new vehicle speed monitoring system, based on an RFID communication.

\section{RFID speed measurement system}

\subsection{Description}

The RFID speed monitoring system, as mentioned above consists of two RFID readers placed at determined distance $\mathrm{D}$, able to get the current information about the vehicle embedded in the transponder and to memorize time of a vehicle passing through the reader interrogation zone. Knowing the times $\Delta t$ and distance travelling between two points we can calculate the speed of each vehicle travelling on road using the basic formula for average velocity (1).

$$
\mathrm{V}=\mathrm{D} / \Delta \mathrm{t}
$$

The model (figure 1) represent side-looking configuration, that have an illuminative direction perpendicular to traffic, this configuration is the most popular approach for current ITS (intelligent transportation systems) applications which can cover all the lanes [12].

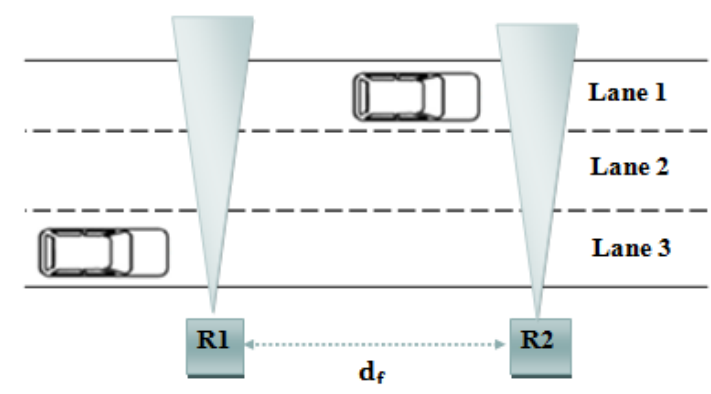

Figure 1: side-looking RFID radar systems.

\subsection{Error analysis}

RFID speed monitoring system has some desirable features, such as low cost, not requiring line of sight between readers and labels to detect the target vehicle. However, such devices suffer from the limited accuracy of average speed especially in the event where the roadway is composed of several lanes and in the presence of multiple vehicles. To insure a well-functioning, it's primordial to study all the system aspects.

One of the main disadvantages is that a reader's log the times which are not adapted to a transmission positions. This notion is also extremely important, because it can easy miss significant changes on travelled distance $\mathrm{D}$, which can provide error in speed measurement. In other words the speed error provided is caused by a shift position between the reference axis and the line connection to the tag $\left(\Delta \mathrm{d}_{1}, \Delta \mathrm{d}_{2}\right)$; weather it is for the first reader $\mathrm{R} 1$, or the second reader R2 (Figure 2).

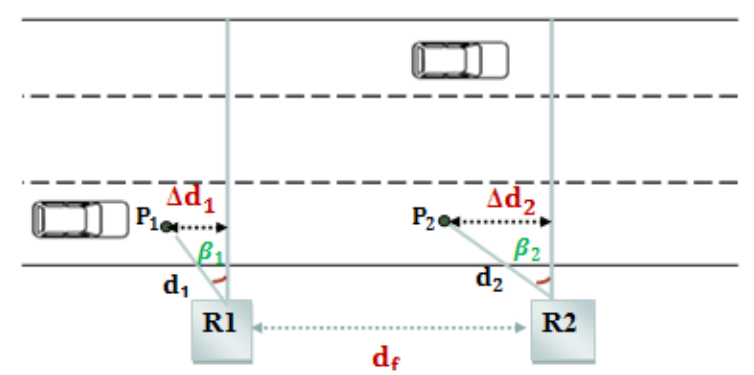

Figure 2: Components of RFID speed measurement system.

This paper aims to determine the impact of all parameters contributing to the RFID speed measurement system and their impact on speed accuracy. 


\section{THE SPEED-ACCURACY}

The first stage of this work will be the determination of all factors needed to calculate speed. We'll start by expressing the travelled distance $\mathrm{D}$ by the target with reference to figure (1):

$$
\mathrm{D}=\mathrm{d}_{\mathrm{f}}+\Delta \mathrm{d}_{1}-\Delta \mathrm{d}_{2}
$$

Where $d_{f}$ is the distance between two readers, and $\Delta \mathrm{d}_{1}, \Delta \mathrm{d}_{2}$ are the maximum error to detecte the position of transponder by the first and second reader respectively, with :

$$
\begin{aligned}
& \Delta \mathrm{d}_{1}=\operatorname{Sin}\left(\beta_{1}\right) * \mathrm{~d}_{1} \\
& \Delta \mathrm{d}_{2}=\operatorname{Sin}\left(\beta_{2}\right) * \mathrm{~d}_{2}
\end{aligned}
$$

Where $d_{1}, d_{2}$ are the distances between the readers and tag attached to vehicles, $\beta_{1}, \beta_{2}$ are the angle between the reference axis and the line connection with the tag, we can express :

$$
\beta_{2}=\beta_{1} \pm \varepsilon
$$

Where $\varepsilon$ the variation of detection angle by the two readers. According to Friis Transmission Equation we can obtain [13] [14]:

$$
\left(\mathrm{d}_{1}, \mathrm{~d}_{2}\right)=\frac{\lambda}{(4 \pi)} \sqrt{\mathrm{P}_{\mathrm{EIRP}} \mathrm{G}^{\mathrm{Tag}} \tau_{\text {tag }} \chi \frac{1}{\mathrm{P}_{\mathrm{R}}^{\text {Chip }}}}
$$

Where $\mathrm{P}_{\text {EIRP }}$ The maximum power output available for RFID applications, $G^{\text {Tag }}$ is the gain of the tag antenna respectively, $\tau_{\text {tag }}$ is the reflection coefficient between the tag antenna and the chip, $\chi$ is the polarization factor between reader antenna and tag antenna, $\lambda$ wavelength and $\mathrm{P}_{\mathrm{R}}^{\text {Chip }}$ is received power at the chip.

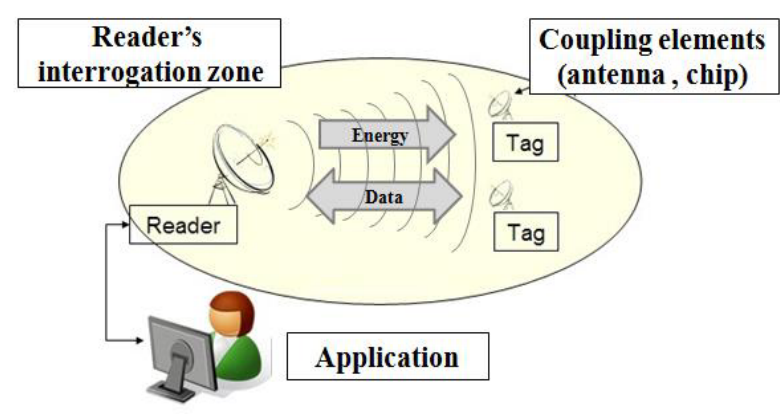

Figure 3: RFID Communication.

Figure 3 illustrates the standard communication between an RFID tag and an RFID reader. The reader transmits an interrogation signal which energizes and activates the RFID tag. Then the tag detects this signal, it responds and is identified [15]. For simplification only a single transponder considered present in the interrogation zone, however if several tags are simultaneously in the area covered by the reader, it will be necessary to integrate collision avoidance algorithms to ensure reading all these transponders [16]. The expression of the $\Delta d_{1}$ and $\Delta d_{2}$ is given in Equation (7) and (8) according to equation (6):

$$
\begin{aligned}
& \Delta \mathrm{d}_{1}=\operatorname{Sin}\left(\beta_{1}\right) \frac{\lambda}{(4 \pi)} \sqrt{\left(\mathrm{P}_{\mathrm{EIRP}} \mathrm{G}^{\mathrm{Tag}} \tau_{\mathrm{tag}} \chi \frac{1}{\mathrm{P}_{\mathrm{R}}^{\text {Chip }}}\right.} \\
& \Delta \mathrm{d}_{2}=\operatorname{Sin}\left(\beta_{1}+\varepsilon\right) \frac{\lambda}{(4 \pi)} \sqrt{\left(\mathrm{P}_{\mathrm{EIRP}} \mathrm{G}^{\mathrm{Tag}} \tau_{\text {tag }} \chi \frac{1}{\mathrm{P}_{\mathrm{R}}^{\text {Chip }}}\right.} \\
& V=\frac{d_{f}+\Delta d_{1}-\Delta d_{2}}{T} \\
& \mathrm{~V}=\frac{\mathrm{d}_{\mathrm{f}}+\left(\frac{\lambda}{(4 \pi)} \sqrt{\left(\mathrm{P}_{\mathrm{EIRP}} \mathrm{G}^{\mathrm{Tag}_{\operatorname{tag}_{\mathrm{tag}}}{ }_{\mathrm{P}_{\mathrm{R}} \frac{1}{\text { Chip }}}}\right) *\left(\operatorname{Sin}\left(\beta_{1}\right)-\operatorname{Sin}\left(\beta_{1}+\varepsilon\right)\right)}\right.}{\mathrm{T}}
\end{aligned}
$$

\section{RESULTS AND DISCUSSION}

Furthermore, we study the influence of system parameters on the measurement error generated by speed computing. The first parameter to be analysing is $d_{f}$, in order to determine the optimum choice for this factor. Speed Error curve is plotted according to equation (12) by substituting different $\Delta \mathrm{d}_{1}$ values and using the values listed below in Table 1.

$$
\begin{aligned}
& \text { Error speed }(\%)=\frac{\text { Speed }_{\text {Approah }}-\text { Speed }_{\text {sensed }}}{\text { Speed }_{\text {sensed }}} * 100 \\
& \text { Error speed }(\%)=\left|\frac{\left({ }^{d_{f}} / T\right)-\left({ }^{d_{f}}+\Delta d_{1}-\Delta d_{2} / T\right)}{\left(d_{f}+\Delta d_{1}-\Delta d_{2} / T\right)}\right| * 100
\end{aligned}
$$

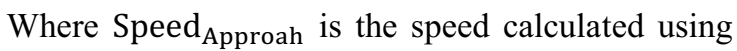
$\mathrm{D}$ traveled by target equal to distance a fixed distance of readers $\left(D=d_{f}\right)$, then Speed $_{\text {sensed }}$ is calculated by using the equation (10).

Table 1: Parameters used in curves.

\begin{tabular}{|c|c|}
\hline Parameters & Values \\
\hline $\mathrm{T}(\mathrm{s})$ & 1 \\
\hline$\Delta \mathrm{d}_{2}(\mathrm{~m})$ & 4 \\
\hline
\end{tabular}

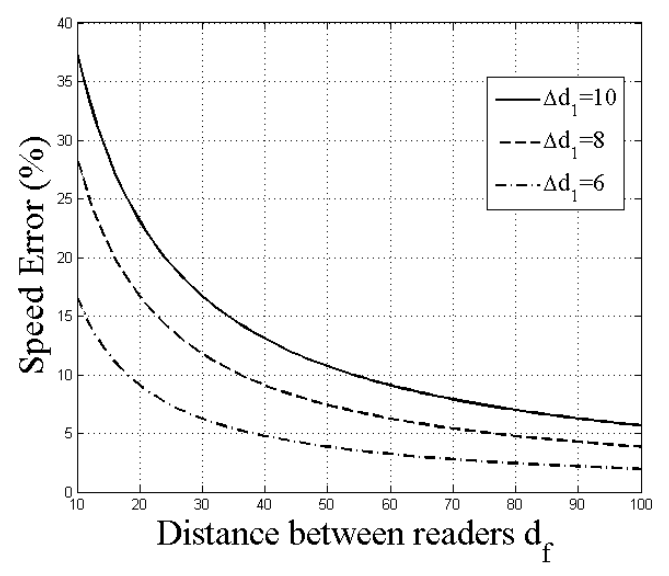

Figure 4: Speed error as function of distance between readers $\mathrm{d}_{\mathrm{f}}$ for differentshift position $\Delta \mathrm{d}_{1}(\mathrm{~m})$. 
As shown, the increasing distance between two readers, will decrease the speed error for different values of variation in detection $\Delta \mathrm{d}_{1}$. However, the placement of the two readers at far distance may lead to erroneous results, due to changing lanes or decrease the speed between two consecutive readers. To avoid this type of error we assume that the minimum distance between two readers can be expressed by:

$$
\mathrm{d}_{\mathrm{f}}=2 * \mathrm{R} * \sin (\Theta)+\mathrm{s}
$$

Where the $\Theta$ is the half aperture angle of reader's antenna, $\mathrm{R}$ is maximal read range (14) and $\mathrm{s}$ is the security distance to avoid the interference between the reader's interrogations zone (figure 5) which can we take $10 \%$ of $\mathrm{R}$.

$$
\begin{gathered}
R=\frac{\lambda}{(4 \pi)} \sqrt{\mathrm{P}_{\mathrm{EIRP}} \mathrm{G}^{\mathrm{Tag}} \tau_{\text {tag }} \chi \frac{1}{\mathrm{P}_{\mathrm{Rmin}}^{\text {Chip }}}} \\
\mathrm{d}_{\mathrm{f}}=\frac{\lambda}{(4 \pi)} \sqrt{\mathrm{P}_{\mathrm{EIRP}} \mathrm{G}^{\mathrm{Tag}} \tau_{\text {tag }} \chi \frac{1}{\mathrm{P}_{\mathrm{R} \min }^{\text {Chip }}}} *(2 * \sin (\Theta)+0.1)
\end{gathered}
$$

With $\mathrm{P}_{\mathrm{Rmin}}^{\mathrm{Chip}}$ is the threshold power of a transponder. In case where the two readers have the same characteristics and the width of the road varies from 14 to 16 meters, the distance $d_{f}$ won't exceed the 32 meters. Unfortunately, this distance become $\mathrm{d}_{\mathrm{f}}=32$, according to the figure (4), this value tend to provide significant error.

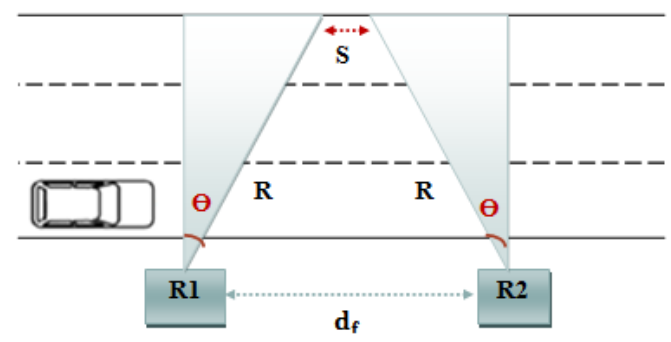

Figure 5: $\mathrm{d}_{\mathrm{f}}$ parameters.

According to the equation (10) we have noted that the speed depends on other parameters of the transponder. In this effect we sought to determine the impact of each one (12). Using table 2, we obtain the impact of some parameters in the speed measurement

Table 2: Parameters Used in Simulation.

\begin{tabular}{|c|c|}
\hline Parameters & Values \\
\hline $\begin{array}{c}\text { Equivalent Isotropic } \\
\text { Radiated Power }\end{array}$ & $500 \mathrm{~mW}$ ERP \\
\hline Polarization factor & $-3 \mathrm{~dB}$ \\
\hline Tag power sensitivity & $-35 \mathrm{dBm}$ \\
\hline
\end{tabular}

\begin{tabular}{|c|c|}
\hline$\Theta$ & $40^{\circ}$ \\
\hline$\beta_{1}$ & $15^{\circ}$ \\
\hline $\mathrm{T}(\mathrm{s})$ & 1 \\
\hline
\end{tabular}

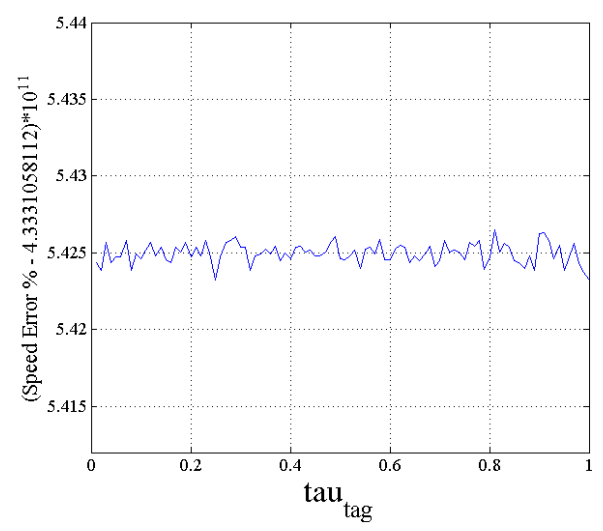

Figure 6: relative speed error as a function of the reflexion coefficient. Other parameters: $867.2 \mathrm{MHz}$, $\mathrm{G}^{\mathrm{Tag}}=2 \mathrm{dBi}, \mathrm{P}_{\mathrm{R}}^{\mathrm{Chip}}=-25 \mathrm{dBm}, \varepsilon=10^{\circ}$.

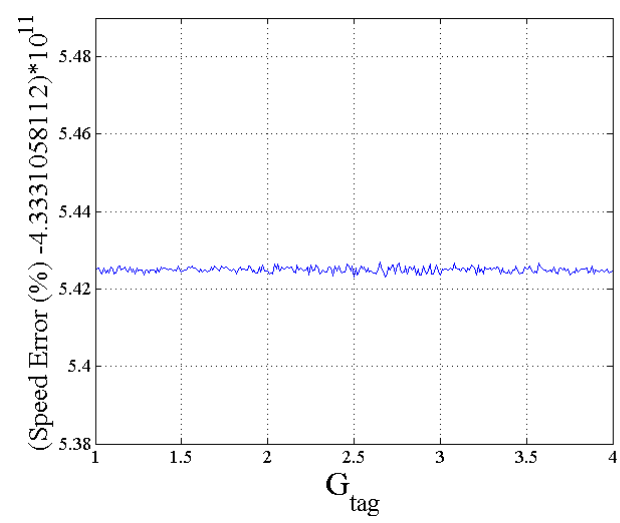

Figure 7 : Speed error as a function of the gain $(\mathrm{dBi})$. Other parameters: $867.2 \mathrm{MHz}, \tau_{\text {tag }}=0.8, \mathrm{P}_{\mathrm{R}}^{\text {Chip }}=$ $-25 \mathrm{dBm}, \mathcal{E}=10^{\circ}$.

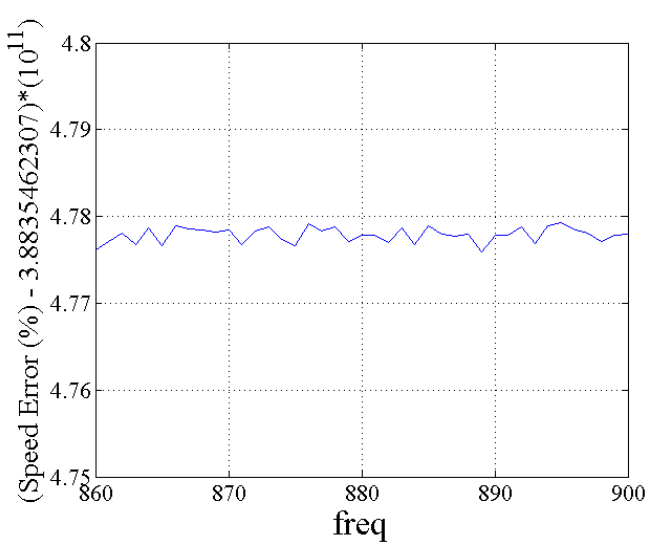

Figure 8: Speed error as a function of frequency $(\mathrm{MHz})$. Other parameters, $\mathrm{G}^{\mathrm{Tag}}=2 \mathrm{dBi}, \tau_{\mathrm{tag}}=0.8, \mathrm{P}_{\mathrm{R}}^{\text {Chip }}=-25 \mathrm{dBm}$, $\varepsilon=10^{\circ}$. 
The figure (6,7and 8) shows a slight error occurs by these parameters (gain $\mathrm{G}^{\mathrm{Tag}}$, reflexion coefficient $\tau_{\text {tag }}$, frequency) Generally, we can note that the error effecting by these parameters can be imperceptible and can be neglected.

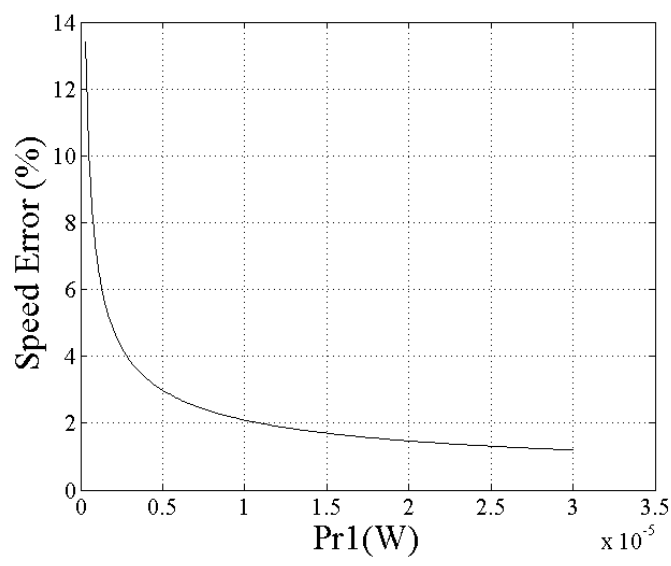

Figure 9: Speed error as a function received power at the chip. Where $867.2 \mathrm{MHz}, \mathrm{G}^{\mathrm{Tag}}=2 \mathrm{dBi}, \tau_{\mathrm{tag}}=0.8, \varepsilon=10^{\circ}$

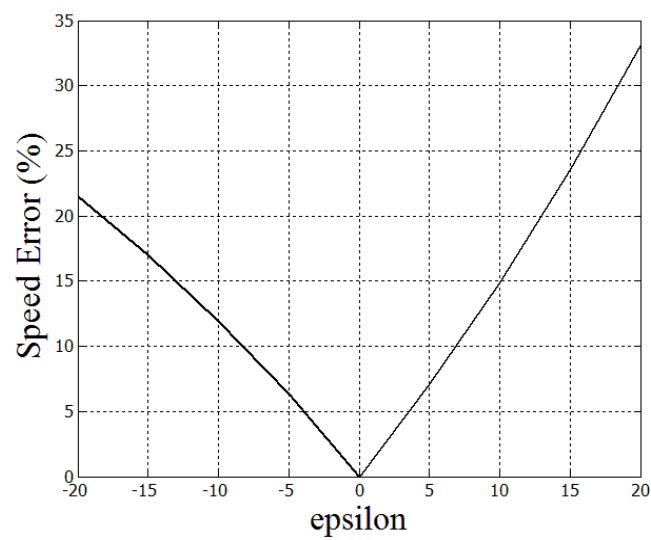

Figure 10 :Speed error as a function of $\varepsilon$, where867.2 $\mathrm{MHz}, \mathrm{G}^{\mathrm{Tag}}=2 \mathrm{dBi}, \tau_{\mathrm{tag}}=0.8, \mathrm{P}_{\mathrm{R}}^{\mathrm{Chip}}=-25 \mathrm{dBm}$.

The figure (9) presents the impact of received power at the chip on speed error. The error grows with decreasing received power at the chip $\left(\mathrm{P}_{\mathrm{R}}^{\mathrm{Chip}}\right)$ due to the fact that the parameter mentioned is the main factor which control the distance between reader and target. Hence, the error grows when the target is so far to the reader with the narrow opening angle or wide opening angle. So, we can say that the power received by the chip is inversely proportional to the speed error. The figure 10 reveals with the increasing $\varepsilon$ the variation of detection angle the speed error increase.

However the error speed provided by the RFID Speed measurement system is related in the first hand to the parameters $\varepsilon$, and the second hand to received power at the chip $\mathrm{P}_{\mathrm{R}}^{\text {Chip }}$.So, in order to compute and have trusted information regarding the speed, we intend to normalize the $\varepsilon$ value such a way that the margin of error or tolerance must not exceed $5 \%$ comparatively to speedometers existing.

\section{Conclusion \& future works}

This paper gives in the first hand a comparative overview of vehicle speed measurement systems, and then we focus especially on new RFID speed measurement system. In the second hand, we presented the method used to calculate average speed by this system by referring to a model of communication RFID. In fact this work is principally concerned with the parameters that influencing on vehicle speed measurement using RFID technology.

The power received at the chip and the variation of detection angle are the key factors affecting the accuracy of the average speed.

As a future work we are working towards to reducing the speed error by normalize the variation of angle detection, and to find the minimal opening angle of RFID reader antenna, to concentrate the power toward each tags, while insure the read of tags embedded in vehicle driving at high speed. Afterwards we intend to find a model of tag specific applicable RFID speed measurement system.

\section{References}

1. B. Bakama Nume, Road Traffic Accidents in Uganda: Epidemiological and Transport Policy Implications(African Social Science Review, 2006).

2. M. Peden, R. Scurfield, D. Sleet, Di. Mohan, A. Hyder, E. Jarawan and C. Mathers, World report on road traffic injury prevention (World Health Organization Geneva 2004).

3. O. Bagadadi and A. Varhelyi, An indicator of accident proneness (Accident Analysis and Prevention, vol. 43, pp. 1359-136, 2011).

4. Institute of safety road, The relation between speed and crashes(SWOV Fact sheet, April 2012).

5. T. Litman, S. Fitzroy, Evaluating Mobility Management Traffic Safety Impacts (Victoria Transport Policy Institute, 12 September 2016).

6. S. Park, T. Kim, S. Kang, and K. Heon Koo, $A$ Novel Signal Processing Technique for Vehicle Detection Radar, (EEE MIT-S Digest, 2003).

7. Mphippen, Difference Between Radar and Lidar Explained, (IPTM Special Problems in 
Traffic Crash Reconstruction Conference, May 2013).

8. M. Ayub Khan, S. Ziauddin, I. Haq, N. Mehmood, M. Hussain, S. Hassan, M. Tayyab, Apparatus and method for automatic license plate recognition and traffic surveillance, US20150248595 A1,3 sept. 2015

9. W. Wencheng, Edgar A. Bernal, Robert P. Loce, Thomas F. Wade, Abu Islam, Single camera video-based speed enforcement system with a secondary auxiliary $R G B$ traffic camera, US9183746 B2,nov 2015.

10. K. Park, T. Kang, S. Hyun, S. Kang, B. Choi, Method for measuring speed of vehicle using RFID, RFID reader for measuring speed of vehicle, and system for collecting vehicle information using the same,US8274371 B2,25 sept. 2012.

11. A. Al-Gindy, A. Tawfik, L. Sakhi , RFID Speed monitoring system , (Sixth International Conference on Developments in eSystems Engineering, 2013).

12. S. Jeng, W. Chieng, and L. Hsiang-Pin, Estimating Speed Using a Side-Looking Single-Radar Vehicle Detector (IEEE transactions on intelligent transportation systems, vol. 15, no. 2, april 2014).

13. M. Dhaouadi, Conception et optimisation des antennes RFID UHF en vue d'améliorer la fiabilité des systèmes RFID (2014).

14. P. Jankowski-Mihulowicz, M. Weglarsk, Determination of Passive and Semi-Passive Chip Parameters Required for Synthesis of Interrogation Zone in UHF RFID Systems, (ELEKTRONIKAIR ELEKTROTECHNIKA, ISSN 1392-1215, VOL. 20, NO. 9, 2014).

15. H. Peter Cole, Zhonghao $\mathrm{Hu}$ and Y. Wang, Operating Range Evaluation of RFID Systems (The University of Adelaide, Australia).

16. D. Klair, K. Chin, R. Raad, A survey and tutorial of RFID anti-collision protocols (IEEE Communications Surveys \& Tutorials, vol.12, (3) pp. 400-421, 2010). 